\title{
Emotional intelligence in medical students: should we be assessing this more vigorously during the admissions process?
}

\author{
Faiz Ahmed Shaikh and Jenna Gilmour \\ GKT School of Medicine, King's College London, London, UK
}

As fourth-year medical students we read, with great interest, the article titled "The relationship between emotional intelligence and happiness in medical students" by Ghahramani et al. [1] and gained an appreciation for the insight into the importance of the mental health of medical students and how this can potentially impact their practice. It is therefore plausible to consider whether the ideology of emotional intelligence should be more vigorously tested during the medical school admissions process.

Based on our experience, we agree that emotional intelligence is multifactorial but we have found that it often manifests itself as a learning curve with a typical pattern of progression during medical school. If we focus on empathy, we found that during first year of medical school with limited clinical exposure and knowledge, there are higher levels of empathy as a substitute for lack of clinically oriented questioning. However, with progression through medical school, the increase in clinical knowledge is often accompanied by a negative correlation in empathy levels [2]. An example of this is during history taking whereby in preclinical years, with no structure or expectation of making differential diagnoses, our conversation pertained more to the patients' ideas, concerns, and expectations and made for a more genuine conversation between two individuals. However, this rapport soon dissipated in early clinical years as we were confined to a structure and asked relevant questions regarding things like family history and the cause of their parents' death as opposed to the impact this had on that patient.

As our clinical knowledge grows and our structured approach to both history taking and examination becomes instinctive and requires less conscious thought, you rediscover what it is to care for your patients rather than treating them as a differential diagnosis, after all, we treat the patient, not the disease.

This pattern can be likened to an inverted bell-shaped curve and has been brought to light through selfreflection as well as seeking feedback from more experienced colleagues. With this being said, an important factor to consider is the baseline level of
Received: May 7, 2019 • Revised: May 14, 2019 • Accepted: May 15, 2019 Corresponding Author: Faiz Ahmed Shaikh (https://orcid.org/0000-0001-6340-3702) GKT School of Medicine, King's College London, Guy's Campus, Great Maze Pond, London, UK Tel: +44.07553883993 email: k1328995@kcl.ac.uk
Korean J Med Educ 2019 Jun; 31(2): 177-178.

https://doi.org/10.3946/kjme.2019.129

eISSN: 2005-7288

(C) The Korean Society of Medical Education. All rights reserved. This is an open-access article distributed under the terms of the Creative Commons Attribution Non-Commercial License (http:// creativecommons.org/licenses/by-nc/3.0/), which permits unrestricted non-commercial use, distribution, and reproduction in any medium, provided the original work is properly cited. 
empathy with respect to emotional intelligence of new medical students [3], which we feel is often overshadowed during the admissions process.

As found in your study, the biggest predictor of happiness was personality types, which then goes on to predict the level of emotional intelligence amongst medical students. The admissions process for medicine, focusses more on extra-curricular achievements and aptitude scores in order to select successful candidates as high academic grades are a given. Although the introduction of multiple mini interviews at some medical schools [4] provides an opportunity to assess the emotional intelligence of candidates [5], we feel that they still do not sufficiently delve into their personality type, which begs the question whether the admissions process needs to improve to incorporate more predictors of emotional intelligence such as self-awareness, self-control, and emotional readiness as these skills ultimately mould a medical student into a good and 'happy' doctor thus reinforcing their role in improving community health.

In conclusion, "The relationship between emotional intelligence and happiness in medical students" has provided an insight to the importance of emotional intelligence amongst medical students [1] and how currently the admissions process is somewhat lacking in assessing this crucial aspect of a future clinicians personality. Therefore, medical educators should look to improve the admissions process and focus more on a candidate's emotional readiness to study medicine to ultimately produce more caring doctors.

\section{ORCID:}

Faiz Ahmed Shaikh: https://orcid.org/0000-0001-6340-3702;
Jenna Gilmour: https://orcid.org/0000-0002-1718-9562

Acknowledgements: None.

Funding: None.

Conflicts of interest: No potential conflict of interest relevant to this article was reported.

Author contributions: Study conception and design: FAS, JG; research of literature: FAS; analysis and interpretation of literature: FAS, JG; drafting of the manuscript: FAS; and critical revision: FAS.

\section{References}

1. Ghahramani S, Jahromi AT, Khoshsoroor D, Seifooripour $\mathrm{R}$, Sepehrpoor M. The relationship between emotional intelligence and happiness in medical students. Korean J Med Educ. 2019;31(1):29-38.

2. Mintle LS, Greer CF, Russo LE. Longitudinal assessment of medical student emotional intelligence over preclinical training. J Am Osteopath Assoc. 2019;119(4):236-242.

3. Irfan M, Saleem U, Sethi MR, Abdullah AS. Do we need to care: emotional intelligence and empathy of medical and dental students. J Ayub Med Coll Abbottabad. 2019;31(1):76-81.

4. Knorr M, Schwibbe A, Ehrhardt M, Lackamp J, Zimmermann S, Hampe W. Validity evidence for the Hamburg multiple mini-interview. BMC Med Educ. 2018;18(1):106.

5. Dolev N, Goldental N, Reuven-Lelong A, Tadmor T. The evaluation of emotional intelligence among medical students and its links with non-cognitive acceptance measures to medical school. Rambam Maimonides Med J. 2019;10(2). https://doi.org/10.5041/RMMJ.10365. 